\title{
RELATIVE FREE ENERGY AND ITS APPLICATION TO SPEED CHANGE MODEL
}

\author{
By \\ Makoto Mori and Masasi KowAdA
}

\section{$\S 1$. Introduction.}

The purpose of this paper is to study a continuous spin system by introducing the relative free energy* which plays a role of the free energy in discrete spin systems.

Although the usual free energy is a very important functional, it can be available to only identically distributed 2-spin lattice systems. About such systems, refer to Holley [1], [2]. On the other hand, our relative free energy enables us to deal with spin systems with state space not necessarily discrete nor identically distributed. In Section 3, we consider a 1-dimensional lattice spin system with continuous state space to show that the relative free energy does not increase with time and especially it strictly decreases if the initial state is not Gibbsian; this is one of the main results of this paper. From this fact, moreover we conclude that the time evolution of any shift invariant non-Gibbsian state converges to an equilibrium state. The precise definitions of the relative free energy et al are given in Section 2.

\section{$\S 2$. Definition of relative free energy.}

Let $\Omega_{*}$ be a compact Hausdorff space with Second countability axiom, and let $\mathscr{B}_{*}$ be its topological Borel field. We suppose that a probability measure $\nu_{*}$ is given on $\left(\Omega_{*}, \mathscr{B}_{*}\right)$. We denote the two sided countable direct product of copies of $\left(\Omega_{*}, \mathscr{B}_{*}, \nu_{*}\right)$ by $(\Omega, \mathscr{B}, \nu)$. Let $C_{n}$ be the family of all $(-n, n)$-cylinder sets $i$. e. the sets of the form $\left\{\omega \in \Omega ; \omega_{i} \in E_{i}, i=0, \pm 1, \cdots, \pm n\right\}, E_{i} \in \mathscr{B}_{*}$, and we denote by $\mathscr{B}_{n}$ the $\sigma$-algebra generated by $C_{n}$.

The restriction to $\mathscr{B}_{n}$ of a probability measure $\mu$ on $\mathscr{B}$ is denoted by $\mu^{(n)}$. Moreover the set of all probability measures on $\mathscr{B}\left(\mathscr{B}_{n}\right)$ is denoted by $\mathscr{P}\left(\mathscr{P}_{n}\right)$.

Received November 20, 1979. Revised November 28, 1980.

* In [5], Sulivan defined the relative free energy which is similar to ours and obtained the similar results. But his free energy depends on the special kind of invariant measure of the generator. Therefore it is hardly possible to deal with our model. 
Any measurable finite partition $\xi_{0}$ of $\Omega_{*}$ induces a measurable finite partition $\xi$ of $\Omega$ each atom of which has the form $\left\{\omega \in \Omega ; \omega_{0} \in I\right\}, I \in \xi_{0}$ and we say that $\xi$ is induced by $\xi_{0}$. The family of all measurable finite partitions of $\Omega_{*}$ is denoted by $\Lambda$ and we identify the partition $\xi_{0}$ of $\Omega_{*}$ and the partition $\xi$ of $\Omega$ which is induced by $\xi_{0}$.

Definition 1. For $\mu \in \mathscr{P}$ and any finite measurable partition $\xi$ of $\Omega$, we put

$$
\begin{gathered}
H^{\nu}(\mu, \xi)=\sum_{I \in \xi} \mu(I) \log \frac{\mu(I)}{\nu(I)} \\
h^{\nu}(\mu, \xi)=\varlimsup_{n \rightarrow \infty} \frac{1}{2 n+1} H^{\nu}\left(\mu, \bigvee_{j=-n}^{n} T^{j} \xi\right),
\end{gathered}
$$

where $T$ is the shift transformation; $(T \omega)_{i}=\omega_{i+1}$. In (2.1), we take

$$
\begin{aligned}
& 0 \log 0=0 \\
& p \log \frac{p}{0}= \begin{cases}0 & \text { if } p=0 \\
+\infty & \text { if } p>0,\end{cases}
\end{aligned}
$$

if they appear. Note that $H^{\nu}(\mu, \xi) \geqq 0$, since

$$
\Sigma \mu(I) \log \frac{\mu(I)}{\nu(I)} \geqq \Sigma \mu(I)\left(1-\frac{\nu(I)}{\mu(I)}\right)=0
$$

The following Lemma 1 is a repetition of usual entropy calculus and we omit the proof.

LEMMA 1. Let $\xi<\eta$. Then

$$
H^{\nu}(\mu, \xi) \leqq H^{\nu}(\mu, \eta)
$$

and

$$
h^{\nu}(\mu, \xi) \leqq h^{\nu}(\mu, \eta)
$$

LEMMA 2. If $\mu$ is shift invariant, then

$$
h^{\nu}(\mu, \xi)=\sup \frac{1}{2 n+1} H^{\nu}\left(\mu, \bigvee_{j=-n}^{n} T^{j} \xi\right)
$$

for any $\xi \in \Lambda$.

Proof. We need only to show that $a_{n}=H^{\nu}\left(\mu, \bigvee_{j=0}^{n} T^{j} \xi\right)$ is subadditive, i. e. $a_{n+m} \geqq a_{n}+a_{m}$. When there exists $n$ such that $H^{\nu}\left(\mu, \bigvee_{j=0}^{n} T^{j} \xi\right)=+\infty$, it is trivial to see it. When $H^{\nu}\left(\mu, \bigvee_{j=0}^{n} T^{j} \xi\right)<+\infty$ for any $n$, we get 
Relative free energy and its application to speed change model

$$
\begin{aligned}
& a_{m}+a_{n}-a_{m+n} \\
& =\sum \mu\left(I_{0} \cap \cdots \cap I_{m+n+1}\right) \log \frac{\mu\left(I_{0} \cap \cdots \cap I_{n}\right)}{\nu\left(I_{0} \cap \cdots \cap I_{n}\right)} \cdot \frac{\mu\left(I_{n+1} \cap \cdots \cap I_{m+n+1}\right)}{\nu\left(I_{n+1} \cap \cdots \cap I_{m+n+1}\right)} \\
& \quad \times \frac{\nu\left(I_{0} \cap \cdots \cap I_{m+n+1}\right)}{\mu\left(I_{0} \cap \cdots \cap I_{m+n+1}\right)} \\
& =-\sum \mu\left(I_{0} \cap \cdots \cap I_{m+n+1}\right) \log \frac{\mu\left(I_{0} \cap \cdots \cap I_{m+n+1}\right)}{\mu\left(I_{0} \cap \cdots \cap I_{n}\right) \mu\left(I_{n+1} \cap \cdots \cap I_{m+n+1}\right)} \leqq 0,
\end{aligned}
$$

where $I_{j}=\left\{\omega \in \Omega ; \omega_{j} \in E\right\}$, for some $E \in \xi_{0}$ and $\Sigma$ is taken over all $I_{0}, \cdots, I_{m+n-1}$. Let $U$ be a continuous function on $\Omega_{*} \times \Omega_{*}$ and put

$$
U_{n}(\omega)=\sum_{i=-n}^{n-1} U\left(\omega_{i}, \omega_{i+1}\right), \quad \omega \in \Omega .
$$

Definition 2. For $\mu \in \mathscr{P}$ and $\xi \in \Lambda$, we put

$$
f_{U}^{\nu}(\mu, \xi)=\varlimsup_{n \rightarrow \infty} \frac{1}{2 n+1}\left[\int_{\Omega} U_{n}(\omega) \mathrm{d} \mu^{(n)}(\omega)+H^{\nu}\left(\mu, \bigvee_{j=-n}^{n} T^{j} \xi\right)\right] .
$$

Then the relative free energy $f_{U}^{\nu}(\mu)$ of $\mu$ with respect to $U$ and $\nu$ is defined by

$$
f_{U}^{\nu}(\mu)=\sup _{\xi \in \Lambda} f_{U}^{\nu}(\mu, \xi) .
$$

As can be easily seen, $f_{U}^{\nu}(\mu)=+\infty$ if $\mu^{(n)}$ is not absolutely continuous with respect to $\nu^{(n)}$ for some $n$.

LEMMA 3. If $\mu$ is shift invariant, then

$$
f_{U}^{\nu}(\mu)=\int U\left(\omega_{0}, \omega_{1}\right) \mathrm{d} \mu(\omega)+h^{\nu}(\mu)
$$

where $h^{\nu}(\mu)=\sup _{\xi \in \Lambda} h^{\nu}(\mu, \dot{\xi})$.

DEFINITION 3. We call $h^{\nu}(\mu)$ a relative entropy of $\mu$ with respect to $\nu$.

\section{§ 3. Speed Change Model.}

In this section we consider a speed change model. A physical sketch of our model is as follows;

i) each spin stays in a point with exponential holding time which depends on the nearest neighbour spins,

ii) each spin changes its direction independently with a transition probability $p$.

The process of a speed change model which we are going to deal with is a 
Markov process $\left(\Omega, \mathscr{B},\left\{T_{t}\right\}\right)$ with the infinitesimal generator $\mathcal{G}$ defined by

$$
\mathcal{G} f(\omega)=\sum_{i \in Z} C_{i}(\omega) \int_{\Omega_{*}} p\left(\omega_{i}, \phi\right)\left[f\left(\omega_{i}^{\phi}\right)-f(\omega)\right] \mathrm{d} \nu_{*}(\phi),
$$

where $C_{i}(\omega)=\exp \left[U\left(\omega_{i-1}, \omega_{i}\right)+U\left(\omega_{i}, \omega_{i+1}\right)\right]$ and $\omega_{i}^{\phi} \in \Omega$ is defined by

$$
\left(\omega_{i}^{\phi}\right)_{j}= \begin{cases}\omega_{j} & j \neq i \\ \phi & j=i \quad\left(\phi \in \Omega_{*}\right)\end{cases}
$$

and $p(\phi, \phi)$ satisfies the following four conditions:

i) $L>p(\phi, \phi)>l>0, \quad \phi, \phi \in \Omega_{*}$,

ii) $\int_{\Omega_{*}} p(\phi, \phi) \mathrm{d} \nu_{*}(\phi)=1 \quad$ for any $\phi \in \Omega_{*}$.

iii) $\int \mathrm{d} \nu_{*}(\phi) \int p(\phi, \phi) f(\phi) \mathrm{d} \nu_{*}(\phi)=\int f(\phi) \mathrm{d} \nu_{*}(\phi)$

for any integrable function $f$ on $\Omega_{*}$.

iv) For any $\varepsilon>0$ and any $\phi \in \Omega_{*}$, there exists a neighbourhood $\mathcal{O}_{\psi}$ of $\phi$ such that $\left|p(\phi, \phi)-p\left(\psi^{\prime}, \phi\right)\right|<\varepsilon$ for any $\psi^{\prime} \in \mathcal{O}_{\dot{\psi}}$ and any $\phi \in \Omega_{*}$.

The existence of the Markov process with this generator comes from Ligget [3].

Evidently, the definition domain of $\mathscr{G}$ includes $\mathscr{F}=\bigcup_{n} \mathscr{F}_{n}$, where $\mathscr{F}_{n}$ is the set of all real valued bounded measurable functions which do not depend on the coordinates $\omega_{i}(|i|=n+1, n+2, \cdots)$ of $\omega$. Clearly the generater $\mathcal{G}$ transforms $\mathscr{F}_{n}$ into $\mathscr{I}_{n+1}$.

Let $A_{n, k}=\left\{\left(i_{1}, \cdots, i_{k}\right) ;\left|i_{1}\right| \leqq n,\left|i_{2}\right| \leqq \max \left\{n,\left|i_{1}\right|+1\right\}, \cdots,\left|i_{k}\right| \leqq \max \left\{n,\left|i_{1}\right|\right.\right.$ $\left.+1, \cdots,\left|i_{k-1}\right|+1\right\}$, and let

$$
\mathcal{G}_{(i)}^{*} f(\omega)=\int\left[p\left(\phi, \omega_{i}\right) C_{i}\left(\omega_{i}^{\psi}\right) f\left(\omega_{i}^{\psi}\right)-C_{i}(\omega) f(\omega)\right] \mathrm{d} \nu_{*}(\phi) .
$$

We now define a 'dual operator' $\mathcal{G}_{n}^{* k}(k=1,2, \cdots)$ by

$$
\mathcal{G}_{n}^{* k} f(\omega)=\sum \mathcal{G}^{*}{ }_{\left(i_{k}\right)} \cdots \mathcal{G}^{*}{ }_{\left(i_{1}\right)} f(\omega)
$$

where $f \in \mathscr{F}_{n}$ and the summation is taken over $\left(i_{1}, \cdots, i_{k}\right) \in A_{n, k}$.

The direct calculation gives us the following

$$
\int \mathrm{d} \nu(\omega)\left(\mathcal{G}^{k} f(\omega)\right) g(\omega)=\int \mathrm{d} \nu(\omega) f(\omega) \mathcal{G}_{n}^{* k} g(\omega)
$$

for $f, g \in \mathscr{F}_{n}$. 
Let $L^{1}$ be the space of the integrable functions with respect to $\nu$. Then the the norm estimations of $\mathcal{G} f$ and $\mathcal{G}_{n}^{*} f=\mathcal{G}_{n}^{* 1} f$ are given by,

1) $\|\mathcal{G} f\| \leqq 2(2 n+1) K\|f\|$

2) $\left\|\mathcal{G}_{n}^{*} f\right\| \leqq 2(2 n+1) K\|f\| \quad f \in \mathscr{F}_{n}$,

where $\|\cdot\|$ is the $L^{1}$-norm and $K=L\left(\sup e^{U(x, y)}\right)^{2}$.

LEMMA 4. For $f \in \mathscr{F}$, put

$$
\exp (t \mathcal{G}) f(\omega)=\sum_{k=0}^{\infty} \frac{t^{k}}{k !} \mathcal{G}^{k} f(\omega) .
$$

Then the right term converges in the $L^{1}$-norm and we have

$$
\left.\frac{\mathrm{d}}{\mathrm{d} t} \exp (t \mathcal{G}) f(\omega)\right|_{t=0}=\mathcal{G} f(\omega) \quad \text { a. e. }
$$

Proof. Remark that $C_{i}(\omega) \int p\left(\omega_{i}, \phi\right)\left[f\left(\omega_{i}^{\psi}\right)-f(\omega)\right] \mathrm{d} \nu_{*}(\phi)$ depends only on $\left(\omega_{-n}, \cdots, \omega_{n}\right)$ for $i=0, \pm 1, \cdots, \pm(n-1)$ and that it depends only on $\left(\omega_{-n-1}, \cdots, \omega_{n}\right)$ for $i=-n$ and $\left(\omega_{-n}, \cdots, \omega_{n+1}\right)$ for $i=n$. Appealing to this fact, we get

$$
\|\exp (t \mathcal{G}) f\| \leqq\|f\| \exp [4 K t(2 n+1+\exp (4 K t))]
$$

for $f \in \mathscr{F}_{n}$. As to (3.11) we have

$$
\begin{aligned}
& \left\|\frac{1}{h}\left\{\sum_{k=0}^{\infty} \frac{h^{k}}{k !} \mathcal{G}^{k} f-f\right\}-\mathcal{G} f\right\| \\
& \left.\quad \leqq \frac{1}{h}\left\|\sum_{k=2}^{\infty} \frac{h^{k}}{k !} \mathcal{G}^{k} f\right\| \leqq h \| \sum_{k=0}^{\infty} \frac{h^{k}}{k !} \mathcal{G}^{k}\left(\mathcal{G}^{2}\right) f\right) \| .
\end{aligned}
$$

Thus

$$
\lim _{h \rightarrow 0} \frac{1}{h}\left\|\left\{\sum_{k=0}^{\infty} \frac{h^{k}}{k !} \mathcal{G}^{k} f-f\right\}-\mathcal{G} f\right\|=0 .
$$

We shall define the time evolution $\mu_{t}$ of $\mu \in \mathscr{P}$ by

$$
\mu_{t}(f)=\mu(\exp (t \mathcal{G}) f), \quad f \in \mathscr{F},
$$

which becomes a probability measure on $\mathscr{B}$.

LEMMA 5. Suppose that $\mu^{(n)}$ is absolutely continuous with respect to $\nu^{(n)}$ for any $n$. Then $\mu_{t}^{(n)}$ is also absolutely continuous with respect to $\nu^{(n)}$ for any $n$ and $t \geqq 0$. Moreover,

$$
\frac{\mathrm{d} \mu_{t}^{(n)}}{\mathrm{d} \nu^{(n)}}=E\left[\left(\exp \left(t \mathcal{G}^{*}\right) \rho\right)_{n} \mid \mathscr{B}_{n}\right], \quad \text { a. e. }
$$


where

$$
\left(\exp \left(t \mathcal{G}^{*}\right) \rho\right)_{n}=\sum_{k=0}^{\infty} \frac{t^{k}}{k !} \mathcal{G}_{n+k}^{* k} \rho^{(n+k)}
$$

and

$$
\rho^{(m)}=\frac{\mathrm{d} \mu^{(m)}}{\mathrm{d} \nu^{(m)}}
$$

Proof. For $f \in \mathscr{F}_{n}$,

$$
\begin{aligned}
& \int f \mathrm{~d} \nu^{(n)} E\left[\left(\exp \left(t \mathcal{G}^{*}\right) \rho\right)_{n} \mid \mathscr{B}_{n}\right] \\
& \quad=\int f \mathrm{~d} \nu\left(\exp \left(t \mathcal{G}^{*}\right) \rho\right)_{n} \\
& \quad=\sum_{k=0}^{\infty} \frac{t^{k}}{k !} \int \mathrm{d} \nu \mathcal{G}_{n+k}^{* k} \rho^{(n+k)} f \\
& =\sum_{k=0}^{\infty} \frac{t^{k}}{k !} \int \mathrm{d} \mu \mathcal{G}^{k} f \\
& =\int \mathrm{d} \mu \exp (t \mathcal{G}) f \\
& =\mu_{t}(f) .
\end{aligned}
$$

\section{§4. Decreasing Property of relative free energy.}

In this section, we show the decreasing property of free energy. As easily seen by (3.4), $\nu$ is an invariant measure when the potential $U \equiv 0$ (this is the case when each spin moves independently). Thus it is natural to consider the relative free energy with respect to $\nu$.

THEOREM 1. The relative free energy $f_{U}^{\nu}\left(\mu_{t}\right)$ is monotone decreasing in $t$ for any $\mu \in \mathscr{P}$.

To show the above theorem, we employ an approximation technique.

Since the potential $U$ is continuous, for any $\varepsilon>0$, there exists a partition $\xi \in \Lambda$ such that for any partition $\eta>\xi$,

$$
\begin{gathered}
0 \leqq U(x, y)-\inf _{\substack{x^{\prime} \in A \\
y^{\prime} \in B}} U\left(x^{\prime}, y^{\prime}\right)<\varepsilon \\
\left|p(x, \psi)-p\left(x^{\prime}, \psi\right)\right|<\varepsilon, \quad \text { for any } \phi \in \Omega_{*} \text { and } x^{\prime} \in A,
\end{gathered}
$$

where $A$ and $B$ are atoms in $\eta$ including $x$ and $y$, respectively. We define 
Relative free energy and its application to speed change model

$$
U_{\eta}(x, y)=\inf _{\substack{x^{\prime} \in A \\ y^{\prime} \in B}} U\left(x^{\prime}, y^{\prime}\right), \quad A, B \in \eta, x \in A, y \in B .
$$

Then it follows

$$
f_{U_{\eta}}^{\nu}(\mu) \leqq f_{U}^{\nu}(\mu)<f_{U_{\eta}}^{\nu}(\mu)+\varepsilon \quad \text { for all } \mu \in \mathscr{P}
$$

In order to prove the theorem, it is sufficient to prove the following lemma.

LEMMA 6. For any partition $\zeta>\eta(\zeta \in \dot{\Lambda})$,

$$
f_{U_{\eta}}^{\nu}\left(\mu_{t}, \zeta\right) \leqq f_{U_{\eta}}^{\nu}(\mu, \zeta)+\varepsilon K_{*} t
$$

where $K_{*}=e^{2\|U\|}+e^{6\|U\|-1}\left(1+2 e^{2 \varepsilon} L+2 \varepsilon e^{2 \varepsilon}\right)$.

Indeed from this lemma, it follows

$$
f_{U}^{\nu}\left(\mu_{t}\right) \leqq f_{U}^{\nu}\left(\mu_{t}\right)+\varepsilon \leqq f_{U}^{\nu}(\mu)+\varepsilon\left(K_{*} t+1\right)
$$

and this implies the above theorem.

PROOF OF LEMMA 6. For convenience we use the following notations. For $A \in \bigvee_{-n}^{n} T^{j} \eta$, let $A_{i}$ be the $i$-th projection of $A$, and we define

$$
\begin{aligned}
& U_{\eta}(A)=\sum_{i=-n}^{n-1} U_{\eta}\left(\omega_{i}, \omega_{i+1}\right) \\
& C_{i}^{\eta}(A)=e^{U_{\eta}\left(\omega_{i-1}, \omega_{i}\right)+U_{\eta}\left(\omega_{i}, \omega_{i+1}\right)},
\end{aligned}
$$

where $\omega_{i} \in A_{i}|i| \leqq n$,

(note that the right terms of (4.4) and (4.5) do not depend on the choice of $\omega$ ) and let for $B \in T^{i} \eta, E=\tau_{i}^{B}(A)$ be the set such that

$$
E_{j}= \begin{cases}B & j=i \\ A_{j} & j \neq i .\end{cases}
$$

Moreover we put for $A, B \in \eta$

$$
p(A, B)=\int_{B} \inf _{\phi \in A} p(\phi, \phi) \mathrm{d} \nu_{*}(\phi) .
$$

Since

$$
f_{U_{\eta}}^{\nu}(\mu)=\varlimsup_{n \rightarrow \infty} \frac{1}{2 n+1} \sum_{A}\left\{U_{\eta}(A) \mu_{t}(A)+\mu_{t}(A) \log \frac{\mu_{t}(A)}{\nu(A)}\right\},
$$

we shall evaluate the quantity for any partition $\zeta \in \Lambda(\zeta>\eta)$

$$
\begin{array}{r}
\frac{\mathrm{d}}{\mathrm{d} t}\left\{\sum_{A}\left[U_{\eta}(A) \mu_{t}(A)+\mu_{t}(A) \log \frac{\mu_{t}(A)}{\nu(A)}\right]\right\} \\
=\sum_{A}\left(\frac{\mathrm{d}}{\mathrm{d} t} \mu_{t}(A)\right)\left(U_{\eta}(A)+\log \frac{\mu_{t}(A)}{\nu(A)}\right)
\end{array}
$$




$$
\begin{aligned}
= & \sum_{A}\left(\int \mathrm{d} \mu_{t} \sum_{i} C_{i}(\omega) \int p\left(\omega_{i}, \phi\right)\left[\chi_{A}\left(\omega_{i}^{\phi}\right)-\chi_{A}(\omega)\right] \mathrm{d} \nu_{*}(\phi)\right) \\
& \times \log \frac{\mu_{t}(A)}{\nu(A)} e^{U_{\eta}(A)}-\sum_{i} \sum_{A} \sum_{B}\left(\int_{\tau B_{i}} \mathrm{~d} \mu_{t} C_{i}(\omega) \int_{A_{i}} p\left(\omega_{i}, \phi\right) \mathrm{d} \nu_{*}(\phi)\right. \\
& \left.-\int_{A} \mathrm{~d} \mu_{t} C_{i}(\omega) \int_{B} p\left(\omega_{i}, \phi\right) \mathrm{d} \nu_{*}(\phi)\right) \log \frac{\mu_{t}(A)}{\nu(A)} e^{U_{\eta}(A)},
\end{aligned}
$$

where $\sum_{A}$ means the summation over the sets $A \in \bigvee_{-n}^{n} T^{j \zeta}$ and $\sum_{B}$ means the summation over the sets $B \in T^{i} \zeta$.

Let $A \in C_{n}$ be a set such that $\nu(A)>0$ and $\mu_{t}(A)=0$. Then it follows from (3.10) that for sufficiently small $h>0$,

$$
\mu_{t+h}(A)=\int_{t}^{t+h} \frac{\mathrm{d} \mu_{s}(A)}{\mathrm{d} s} \mathrm{~d} s \leqq\left\|\mathcal{G} \chi_{A}\right\| h,
$$

and hence we get

$$
\begin{aligned}
\frac{\mathrm{d}}{\mathrm{d} t} & \left\{U_{\eta}(A) \mu_{t}(A)+\mu_{t}(A) \log \frac{\mu_{t}(A)}{\nu(A)}\right\} \\
& =\lim _{h \rightarrow 0} \frac{1}{h} \mu_{t+h}(A) \log \frac{\mu_{t+h}(A)}{\nu(A)} e^{U_{\eta}(A)} \leqq 0 .
\end{aligned}
$$

Moreover, if there exists some $i$ and $B$ such that $\mu_{t}\left(\tau_{i}^{B} A\right)>0$, then we can easily show that $-\frac{\mathrm{d}}{\mathrm{d} t}\left\{U_{r}(A) \mu_{t}(A)+\mu_{t}(A) \log \frac{\mu_{t}(A)}{\nu(A)}\right\}=-\infty$. Noticing the above facts, we get

$$
\begin{aligned}
& \frac{\mathrm{d}}{\mathrm{d} t}\left\{\sum_{A}\left[\left[U_{\eta}(A) \mu_{t}(A)+\mu_{t}(A) \log \frac{\mu_{t}(A)}{\nu(A)}\right]\right\}\right. \\
& \leqq \sum_{i=-n}^{n} \sum_{A} \sum_{B} \log \frac{\mu_{t}\left(\tau_{i}^{B}(A)\right) e^{U_{\eta}\left(\tau_{i}^{B}(A)\right)} \nu(A)}{\mu_{t}(A) e^{U_{\eta}(A)} \nu\left(\tau_{i}^{B}(A)\right)} \\
& \times \int_{A} C_{i}(\omega) \mathrm{d} \mu_{t}(\omega) \int_{B} p\left(\omega_{i}, \phi\right) \mathrm{d} \nu_{*}(\phi),
\end{aligned}
$$

where we take $0 \log \frac{p}{0}=0, p \log 0=-\infty \quad(p>0)$, and $-\infty \leqq-\infty$, if they appear.

In the above summation the $n$-th term is bounded above; 
Relative free energy and its application to speed change model

$$
\begin{aligned}
& \sum_{A} \sum_{B \in \eta} \log \frac{\mu_{t}\left(\tau_{n}^{B}(A)\right) e^{U_{\eta}\left(\tau_{n}^{B}(A)\right)} \nu_{*}\left(A_{n}\right)}{\mu_{t}(A) e^{U_{\eta}(A)} \nu_{*}(B)} \\
& \quad \times \int C_{n}(\omega) \mathrm{d} \mu_{t}(\omega) \int_{B} p\left(\omega_{n}, \phi\right) \mathrm{d} \nu_{*}(\phi) \\
& \leqq \\
& \sum_{A} \sum_{B} \mu_{t}(A) \log ^{+} \frac{\mu_{t}\left(\tau_{n}^{B}(A)\right) e^{U_{\eta}\left(\tau_{n}^{B}(A)\right)} \nu_{*}\left(A_{n}\right)}{\mu_{t}(A) e^{\left.U_{\eta}(A)\right)} \nu_{*}(B)} e^{2\|U\|} L \nu_{*}(B) \\
& \leqq K_{0}=L e^{6\|U\|-1},
\end{aligned}
$$

where $\log ^{+} x=\max \{0, \log x\}$. Similarly, we can show the $(-n)$-th term is also bounded above.

As to the main term, we proceed as follows; putting

$$
\sigma_{t, \eta \eta}^{(i)}(E)=\mu_{t}(E) e^{U_{\eta}(E)} / \nu_{*}\left(E_{i}\right),
$$

we get

$$
\begin{aligned}
& \sum_{i=-(n-1)}^{n-1} \sum_{A} \sum_{B} \int_{A} \int_{B} p\left(\omega_{i}, \phi\right) C_{i}(\omega) \mathrm{d} \nu_{*}(\phi) \mathrm{d} \mu_{t}(\omega) \\
& \quad \times \log \frac{\mu_{t}\left(\tau_{i}^{B}(A)\right) e^{U \eta\left(\tau_{i}^{B}(A)\right)} \nu_{*}\left(A_{i}\right)}{\mu_{t}(A) e^{U_{\eta}(A)} \nu_{*}(B)} \\
& \leqq \\
& \quad \sum_{i=-(n-1)}^{n-1} \Sigma^{+} \mu_{t}(A)\left(p\left(A_{i}, B\right)+\varepsilon \nu_{0}(B)\right) \log \frac{\sigma_{t, \eta}^{(i)}\left(\tau_{i}^{B}(A)\right)}{\sigma_{t, \eta}^{(i)}(A)} C_{i}^{\eta}(A)\left(1+2 \varepsilon e^{2 \varepsilon}\right) \\
& \quad+\sum_{i=-(n-1)}^{n-1} \Sigma^{-} \mu_{t}(A) p\left(A_{i}, B\right) \log \frac{\sigma_{t, \eta}^{(i)}\left(\tau_{i}^{B}(A)\right)}{\sigma_{t, \eta}^{(i)}(A)} C_{i}^{\eta}(A) \\
& \leqq \\
& \quad \sum_{i=-n+1}^{n-1} \sum_{A} \sum_{B} \mu_{t}(A) p\left(A_{i}, B\right) C_{i}^{\eta_{i}}(A) \log \frac{\sigma_{t, \eta}^{(i)}\left(\tau_{i}^{B}(A)\right)}{\sigma_{t, \eta}^{(i)}(A)} \\
& \quad+\varepsilon(2 n+1) K_{1},
\end{aligned}
$$

where $\Sigma^{+}\left(\Sigma^{-}\right)$means the summation over $A \in \bigvee_{-n}^{n} T^{i \zeta}$ and $B \in \zeta$ for which $\log \frac{\sigma_{t, \eta}^{(i)}\left(\tau_{i}^{B}(A)\right)}{\sigma_{t, \eta}^{(i)}(A)} \geqq 0(<0)$, respectively, and

$$
K_{1}=e^{6\|U\|-1}\left(1+2 e^{2 \varepsilon} L+2 \varepsilon e^{2 \varepsilon}\right) .
$$

Moreover,

$$
\begin{aligned}
\sum_{A} \sum_{B} \mu_{t}(A) p\left(A_{i}, B\right) C_{i}^{\eta}(A) \log \frac{\sigma_{t, \eta}^{(i)}\left(\tau_{i}^{B}(A)\right)}{\sigma_{t, \eta}^{(i)}(A)} \\
=\sum_{A} \sum_{B} \mu_{t}(A) p\left(A_{i}, B\right) C_{i}^{\eta}(A) F_{0}\left(\frac{\sigma_{t, \eta}^{(i)}\left(\tau_{i}^{B}(A)\right)}{\sigma_{t, \eta}^{(i)}(A)}\right)
\end{aligned}
$$




$$
\begin{aligned}
& +\sum_{A} \mu_{t}(A) \sum_{B}\left(p\left(B, A_{i}\right) \frac{\nu_{*}(B)}{\nu_{*}\left(A_{i}\right)}-p\left(A_{i}, B\right)\right) C_{i}^{\eta}(A) \\
\leqq & \sum_{A} \sum_{B} \mu_{t}(A) p\left(A_{i}, B\right) C_{i}^{\eta}(A) F_{0}\left(\frac{\sigma_{t, \eta}^{(i)}\left(\tau_{i}^{B}(A)\right)}{\sigma_{t, \eta}^{(i)}(A)}\right)+e^{2\|U\|} \varepsilon,
\end{aligned}
$$

where

$$
F_{0}(x)=\log +1-x \leqq 0 .
$$

Combing (4.10), (4.11), (4.13), and (4.14), we get

$$
\begin{aligned}
\frac{\mathrm{d}}{\mathrm{d} t} & \left\{\sum_{A} U_{\eta}(A) \mu_{t}(A)+\sum_{A} \mu_{t}(A) \log \frac{\mu_{t}(A)}{\nu(A)}\right\} \\
& \leqq 2 K_{0}+\varepsilon(2 n+1) K_{*},
\end{aligned}
$$

where $K_{*}=K_{1}+e^{2\|U\|}$, and hence

$$
\begin{aligned}
& \left\{\sum_{A} U_{\eta}(A) \mu_{t}(A)+\sum_{A} \mu_{t}(A) \log \frac{\mu_{t}(A)}{\nu(A)}\right\} \\
& \quad \leqq\left\{\sum_{A} U_{\eta}(A) \mu(A)+\sum_{A} \mu(A) \log \frac{\mu(A)}{\nu(A)}\right\}+2 K_{0} t+\varepsilon(2 n+1) K_{*} t .
\end{aligned}
$$

Deviding the both sides in (4.17) by $2 n+1$ and taking $\varlimsup_{n \rightarrow \infty}$ we obtain

$$
f_{U_{\eta}}^{\nu}\left(\mu_{t}, \zeta\right) \leqq f_{U_{\eta}}^{\nu}(\mu, \zeta)+\varepsilon K_{*} t
$$

This completes the proof of the lemma 6 .

DEFINITION 4. We shall agree to say that a measure $\mu \in \mathscr{P}$ is a Gibbsian measure (with respect to $\nu$ ), if for any $n$ the conditional measure $\mu\left(A ; \omega_{k},|k|>n\right)$ satisfies

$$
\begin{array}{r}
\mu\left(A ; \omega_{k},|k|>n=1 / Z\left(\omega_{-n-1}, \omega_{n+1}\right)\right. \\
\quad \times \int_{A} e^{-U_{n+1}(\omega)} \mathrm{d} \nu^{(n)}\left(\omega_{-n}, \cdots, \omega_{n}\right),
\end{array}
$$

where $A \in C_{n}$ and

$$
Z\left(\omega_{-n-1}, \omega_{n+1}\right)=\int e^{-U_{n+1}(\omega)} \mathrm{d} \nu^{(n)}\left(\omega_{-n}, \cdots, \omega_{n}\right) .
$$

As can be easily seen, $\mu$ is a Gibbsian measure if and only if $\mu^{(n)}$ is absolutely continuous with respect to $\nu^{(n)}$ for any $n$ and there exists a version $\rho^{(n)}(\omega)$ $=\frac{\mathrm{d} \mu^{(n)}}{\mathrm{d} \nu^{(n)}}(\omega)$ such that $e^{U_{n}^{(\omega)}} \rho^{(n)}(\omega)$ depends only on $\omega_{-n}$ and $\omega_{n}$.

LEMMA 7. If $\mu$ is Gibbsian, then $\mu_{t}=\mu\left(\mu_{0}=\mu\right)$. 
Relative free energy and its application to speed change model

ProOF. Since $C_{i}\left(\omega_{i}^{\phi}\right) \rho^{(n)}\left(\omega_{i}^{\phi}\right)=C_{i}(\omega) \rho^{(n)}(\omega)$ a. e. $\nu$ for $|i|<n$ and for any $\phi \in$ $\Omega_{*}$, we get

$$
\mathcal{G}_{n}^{*} \rho^{(n)}(\omega)=0 \quad \text { a. e. } \nu, \text { for any } n=1,2, \cdots
$$

Therefore

$$
\mu_{t}(A)=\int \mathrm{d} \nu \chi_{A} \rho^{(n)}=\mu(A) \quad \text { for any } A \in C_{n}, \quad n=1,2, \cdots .
$$

This completes the proof.

THEOREM 2. Let $\mu$ be a shift invariant, non Gibbsian measure, then $f_{U}^{\nu}\left(\mu_{t}\right)$ satisfies only one of the followings

i) $f_{U}^{\nu}\left(\mu_{t}\right)=+\infty$ for any $t \geqq 0$

ii) There exists $t_{0} \geqq 0$ such that $f_{U}^{\nu}\left(\mu_{t}\right)=+\infty$ for $t<t_{0}$ and $f_{U}^{\nu}\left(\mu_{t}\right)$ is strictly monotone decreasing for $t>t_{0}$, unless $\mu_{t_{0}}$ is Gibbsian.

COROLlARY. If the initial state $\mu$ is shift invariant, non-Gibbsian probability measure with finite free energy, and the evolution $\mu_{t}$ converges to a measure $\bar{\mu}$ in the weak topology, then $\bar{\mu}$ is a Gibbsian measure.

PROOF OF THEOREM 2. It is sufficient to show the decreasing property for shift invariant non-Gibbsian measure $\mu$ with $f_{U}^{\nu}(\mu)<\infty$. Thus we assume that $\mu^{(n)}$ has a density $\rho^{(n)}$ for any $n$. Then, since $\mu$ is not Gibbsian, we can find $n_{0}$ such that for any $n \geqq n_{0}$ there exist $i(|i|<n)$ and $\delta>0$ for which

$$
\nu_{*}^{(2 n+2)}\left\{\left(\omega_{-n}, \cdots, \omega_{n}, \phi\right) ; F_{0}\left(\frac{\rho^{(n)}\left(\omega_{i}^{\phi}\right) C_{i}\left(\omega_{i}^{\phi}\right)}{\rho^{(n)}(\omega) C_{i}(\omega)}\right) \leqq-\delta\right\}>0
$$

holds. Here, we may assume $n_{0}=2^{n_{1}}-1$ for some positive integer $n_{1}$.

Let $\xi_{m}$ be a finite partition of $\Omega_{*}$ such that

$$
\left|U\left(x_{0}, y_{0}\right)-U\left(x_{1}, y_{1}\right)\right|<\frac{1}{m}
$$

and

$$
\left|p\left(x_{0}, \phi\right)-p\left(x_{1}, \phi\right)\right|<\frac{1}{m}
$$

where $A, B \in \xi_{m}, x_{0}, x_{1} \in A, y_{0}, y_{1} \in B$ and $\phi \in \Omega_{*}$. Put

$$
\eta_{m}^{(1)}=\bigvee_{i=-n_{0}}^{n_{0}} T^{i} \xi_{m}
$$

Moreover, we let $\eta_{m}^{(2)}$ be a partition defined by 


$$
\left\{\omega ; \frac{k}{m} \leqq \rho^{\left(n_{0}\right)}(\omega)<\frac{k+1}{m}\right\} \quad k=0,1, \cdots, m^{2}-1
$$

and

$$
\left\{\omega ; \rho^{\left(n_{0}\right)}(\omega) \geqq m\right\}
$$

Put $\eta_{m}^{*}=\eta_{m}^{(1)} \vee \eta_{m}^{(2)}$. Let $\eta_{m} \in \Lambda$ be a patition which satisfies

$$
\mathcal{\nu}^{\left(n_{0}\right)}\left\{\cup\left\{A \backslash \cup\left\{B ; B \in \bigvee_{-n_{0}}^{n_{0}} T^{j} \eta_{m} \text { and } B \subset A\right\} ; A \in \eta_{m}^{*}\right\}<m^{-2},\right.
$$

and for a partition $\eta \geqq \eta_{m}$, define the function $(\mu / \nu)^{m}$ by

$$
\left(\frac{\mu}{\nu}\right)^{m}(\omega)=\frac{\mu(I)}{\nu(I)}, \quad \text { if } \omega \in I \in \bigvee_{-n_{0}}^{n_{0}} T^{j} \eta
$$

Then we get,

$$
\lim _{m \rightarrow \infty} \nu\left\{\omega ;\left|\left(\frac{\mu}{\nu}\right)^{m}(\omega)-\rho^{\left(n_{0}\right)}(\omega)\right| \leqq \frac{1}{m}\right\}=0 .
$$

Hence, combining the above fact and (4.22), we get for sufficiently large $m$ and any $i\left(|i|<n_{0}\right)$

$$
\begin{gathered}
\nu_{*}^{\left(2 n_{0}+2\right)}\left\{\left(\omega_{-n_{0}}, \cdots, \omega_{n_{0}}, \phi\right) ; F_{0}\left(\frac{(\mu / \nu)^{m}\left(\omega_{i}^{\phi}\right) C_{i}\left(\omega_{i}^{\phi}\right)}{(\mu / \nu)^{m}(\omega) C_{i}(\omega)}\right)<-\frac{\delta}{2}\right\} \\
=\nu_{*}^{\left(2 n_{0}+2\right)}\left\{\left(\omega_{-n_{0}}, \cdots, \omega_{n_{0}}, \phi\right) ; \omega \in A \in \bigvee_{j=-n_{0}}^{n_{0}} T^{j} \eta, \phi \in B\right.
\end{gathered}
$$

such that

$$
\left.\tau_{i}^{B}(A) \in \bigvee_{j=-n_{0}}^{n_{0}} T^{j} \eta \text { and } F_{0}\left(\frac{\sigma_{0, \eta}^{(i)}\left(\tau_{i}^{B}(A)\right)}{\sigma_{0, \eta}^{(i)}(A)}\right) \leqq-\frac{\delta}{2}\right\}>0 .
$$

We now define

$$
F_{m, n}\left(\mu_{t}\right)=\sum_{i=-2}^{2 n-1} \sum_{A} \mu_{t}(A) \sum_{B} p\left(A_{i}, B\right) C_{i}^{\eta}(A) F_{0}\left(\frac{\sigma_{t, \eta}^{(i)}\left(\tau_{i}^{B}(A)\right)}{\sigma_{t, \eta}^{(i)}(A)}\right),
$$

where $\sum_{A}$ means the summation over $A \in \bigvee_{j=-2}^{2 n} T^{j} \eta$. By (4.30), it follows that

$$
F_{m, n_{1}}(\mu)<-\delta^{\prime}
$$

for some $\delta^{\prime}>0$ and for sufficiently large $m$.

Appealing to the shift-invariance of $\mu_{t}$ and the concavity of $F_{0}$, we have

$$
F_{m, n}\left(\mu_{t}\right) \leqq 2 F_{m, n-1}\left(\mu_{t}\right)
$$

and hence by (4.14) and the continuity of $F_{m, n_{1}}(\mu)$ in $\mu$, we get 
Relative free energy and its application to speed change model

$$
\begin{gathered}
\frac{\mathrm{d}}{\mathrm{d} t}\left\{\sum_{A} U_{\eta}(A) \mu_{t}(A)+\sum_{A} \mu_{t}(A) \log \frac{\mu_{t}(A)}{\nu(A)}\right\} \\
\leqq F_{m, n}(\mu)+\frac{1}{m}\left(2^{n+1}-1\right) K_{*}+2 K_{0} \\
\leqq-2^{n-n_{1}} \frac{\delta^{\prime}}{2}+\frac{1}{m}\left(2^{n+1}-1\right) K_{*}+2 K_{0}
\end{gathered}
$$

and therefore for sufficiently small $t>0$,

$$
\begin{aligned}
&\left\{\sum_{A} U_{\eta_{m}}(A) \mu_{t}(A)+\sum_{A} \mu_{t}(A) \log \frac{\mu_{t}(A)}{\nu(A)}\right\} \\
& \leqq\left\{\sum_{A} U_{\eta_{m}}(A) \mu(A)+\sum_{A} \mu(A) \log \frac{\mu_{t}(A)}{\nu(A)}\right\} \\
&-2^{n-n_{1}-1} \delta^{\prime} t+\frac{1}{m}\left(2^{n+1}-1\right) K_{*} t+2 K_{0} t .
\end{aligned}
$$

Then dividing the both terms by $2^{n+1}+1$ and taking $\varlimsup_{n \rightarrow \infty}$, we get

$$
f_{U_{\eta}}^{\nu}\left(\mu_{t}, \eta\right) \leqq f_{U_{\eta_{m}}^{\nu}}^{\nu}(\mu, \eta)-2^{-n_{1}-2} \delta^{\prime} t+\frac{1}{m} K_{*} t
$$

Therefore it follows that

$$
f_{U}^{\nu}\left(\mu_{t}\right) \leqq f_{U}^{\nu}(\mu)-2^{-n_{1}-2} \delta^{\prime} t+\frac{1}{m}\left(K_{*} t+1\right) .
$$

This completes the proof.

\section{§5. Concluding remark.}

We can show that the variational principle still holds for the relative free energy. Precisely this means that the equation

$$
\inf _{\mu \in S} f_{U}^{\nu}(\mu)=f_{U}^{\nu}(\bar{\mu})
$$

has a unique solution $\bar{\mu} \in S$, and it must be Gibbsian where $S$ is the set of all shift invariant probability measures on $\Omega$. This result will be shown in the forcecoming paper.

\section{Acknowledgement}

The authors would like to thank to Mrs. Sumiko Koyama and Miss Hideko Sato for their beautiful typewriting. 


\section{References}

[1] Holley, R., Pressure and Helmholtz Free Energy in a Dynamic Model of a Lattice Gas, Proc. of sixth Berkeley Symposium on Prob. and Math. Statistics 1971.

[2] Holley, R., Free Energy in a Markovian Model of a Lattice Spin System, Comm. Math. Phys., 23 (1971), 87-99.

[3] Liggett, T.M., Existence Theorems for Infinite Particle System, Trans. Amer. Math. Soc., 165 (1972), 471-481.

[4] Ollagnier, J. M. and Pinchon, D., Free Energy in Spin-flip Processes is Non-increasing, Comm. Math. Phys., 55 (1977), 29-35.

[5] Sulivan, W.G., Markov Processes for random fields, Communications of the Dublin Institute for Advanced Studies, Series A (Theoretical Physics), No. 23 (1975).

Institute of Mathematics

University of Tsukuba

Sakura-mura Niihara-gun

Ibaraki, 305 Japan

Department of Fine Measurement

Nagoya Institute of Technology

Gokiso-cho, Showa-ku

Nagoya, 466 Japan 